\title{
Applications of the Blockchain Technology in Emergency Crisis Management and Rescue Processes
}

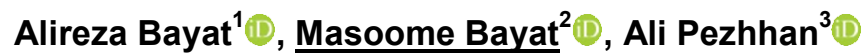

Date of submission: 1 Sep. 2021 Date of acceptance: 14 Sep. 2021

\begin{abstract}
INTRODUCTION: Communication and information exchange are of great importance in order to coordinate crisis management in an emergency and rescue processes. Therefore, the attainment of a strong, complete, and stable communication infrastructure will be among the vital factors for providing an appropriate response to emergencies. The use of new technologies that seem to ensure reliable communication and information exchange must be constantly evaluates.

METHODS: This review study searched articles published from 2000 to 2021 in Persian and English regarding blockchain technology applications in such databases as Google Scholar, Science Direct, and PubMed using keywords, including rescue, blockchain, and crisis management.

FINDINGS: Accurate and optimal presentation of information is an essential prerequisite for crisis management in response to disasters due to the significant impact of each role-maker's decisions. Innovative blockchain technology is extensively used in various fields and is usually well-known and popular for its decentralized and distributed organization and leadership.

CONCLUSION: In general, blockchain technologies have shown success in reducing logistics, support, executive, and management costs in different areas. Crisis management in emergencies and disaster response can be significantly improved by the implementation of blockchain technologies. In addition, studies show that the implementation of blockchain technology in crisis management during an emergency and rescue processes is very complex and requires a great deal of cooperation from all parties. However, it will be effective in practice.
\end{abstract}

Review Article

Keywords: Blockchain; Crisis Management; Rescue.

How to cite this article: Bayat A, Bayat M, Pezhhan A. Applications of the Blockchain Technology in Emergency Crisis Management and Rescue Processes. Sci J Rescue Relief 2021; 13(3): 223-27.

\section{Introduction}

$\mathrm{U}$

nexpected events lead to the death of 150,000 people worldwide each year and cause losses of around $\$ 140$ billion in many countries. Iran, for various reasons, is one of the world's disaster-prone countries, and in the current situation, about $70 \%$ and $50 \%$ of the country is prone to the risk of earthquakes and floods, respectively. Today, it is of significant importance to learn from bitter experiences of the past and consider the unknown future and hazards to prevent unexpected events. Furthermore, in case of confronting such events, it is critical to move toward new crisis management strategies and minimize losses and damages. In addition, the preparedness of "rescue", "treatment", and "support" groups is mandatory in an emergency; otherwise, the unpreparedness of each of these groups will increase casualties, injuries, and damages. It is obvious that new information and communication technologies can help us in all three aforementioned domains (1-3). During an emergency, it is inevitable to utilize new information and communication technologies in order to quickly access the scene of the accident and improve the quality and quantity of rescue. Information and communication

1- Tehran North Branch, Islamic Azad University, Tehran, Iran

2- PhD in Demography, Science and Research Branch, Islamic Azad University, Tehran, Iran

3- Assistant Professor, Department of Social Sciences, Tehran Central Branch, Islamic Azad University, Tehran, Iran

Correspondence to: Masoome Bayat,Email: bayat816@yahoo.com 
technologies are the saviors of humans in difficult situations in which they are not able to solve problems. This technology is a factor that has caused great changes at all social, cultural, and managerial levels and becomes an integral part of modern life. The growth of information and communication technologies in the last ten years has caused human knowledge to proliferate, compared to the last fifty years, and enter new world arenas. In general, the use of new information technologies in the realm of rescue has helped to find the injured more quickly and reduce the rescue time. Regarding healthcare, it leads to patient referral based on available capacities, a decrease in treatment costs, an increase in the efficiency of healthcare, and appropriate notifications. Considering support, the required equipment can be dispatched to the affected areas based on real needs and sufficient information (2, 4-6).

\section{Methods}

This narrative review searched articles from 2000 to 2021 in Persian and English on the applications of blockchain technology through Google Scholar, Science Direct, and PubMed databases using such keywords as rescue, blockchain, and crisis management.

\section{An introduction to the concept of blockchain technology}

"Blockchain" technology is one of the technologies that has gained much attention recently and is undergoing heavy media operations. There are many definitions of this technology. According to developers, it is a set of protocols and encryptions to securely store information on distributed networks. In the realm of economy and business, it is a platform for designing digital currencies, and for engineers, it is a factor to alter the next generation of the Internet. Moreover, it is regarded as a means of transforming society and economics to achieve a decentralized world. The scope of this science is very wide, and this is one of the reasons why blockchain technology has become the greatest human invention after the Internet (7-9). Blockchain is a combination of the words "Block" and "Chain". This technology is a chain of blocks. "Blockchain" technology is a stored general file in which data is stored in batches in well-structured blocks, and each block is connected to the next block using a code. If a block is connected to a chain, the accuracy and authenticity of its data are verified, and a connected and valid chain is created. Therefore, Blockchain is not just a technology rather it is a culture and a community the ultimate goal of which is to achieve a fairer world through decentralization. Furthermore, Blockchain is a type of information and reporting system. The information stored in this system is shared among all members of the network, and it is impossible to delete and manipulate the recorded information using encryption $(10-14,8)$. Blockchain technology is generally considered an important technology that can significantly change business models and processes, as well as the economy (15). Furthermore, this innovative technology is extensively used in a variety of fields, from common processes related to information systems, such as organizational modeling, to specialized topics, including fraud and financial misuse. This indicates that blockchain is a kind of technical and economic innovation (16). The previously conducted studies confirm this hypothesis that one of the important benefits of using blockchain technology is its decentralized organization and distribution, which may lead to increased objectivity and trust (15).

\section{Applications of blockchain technology in medicine and health}

Nowadays, blockchain technology is present in every field of business, and therefore, the healthcare sector has also benefited from this technology. Some features provided by blockchain that are of great interest to healthcare organizations include intermediation, transparency, auditing, industry collaboration, and new business models (17). This technology is going to enter the medical field, and over the past five years, the largest technology companies, such as IBM, have devoted significant resources to comprehensive studies on the blockchain. The results of these studies lead to a better understanding of the applications of this technology in the field of healthcare. According to a study, more than half of the health executives are strongly inclined to have some form of blockchain technology in the medical system in the future since blockchain technology will provide more efficient and safer tools in this area. 
The unchanging nature of this technology can bring about dramatic changes for the healthcare sector, and therefore, it seems perfectly appropriate for this sector (18). All patients' medical records can be recorded together using a global blockchain-based health network. Each patient can have a unique blockchain fingerprint to ensure that his or her identity and medical history are authentic. When this system is used for a large amount of data, health professionals will be well aware that how accurate this new trend can be. In addition, the widely scattered health records can be combined to provide an opportunity to track personal medical history using blockchain $(4,17,18)$. Moreover, various components, such as the time and place of distribution and supply of medications can be more closely monitored. Monitoring of these changes is easily possible through the blockchain, and all results will be immutable (19). Blockchain protects the privacy of user information using its encryption function. Therefore, there is no fear of threats or bias when verifying information on the network. This improves the accuracy of the information so that the right decision can be made at the right time. Information can also be kept secure through the blockchain (6). According to a study conducted by Pereira et al., the combination of artificial intelligence and the blockchain could lead to solutions to health care problems. However, in addition to technical barriers, such as access to and storage of data in the blockchain, there are barriers in policy and privacy issues (20).

\section{Applications of blockchain technology in emergency crisis management and rescue processes}

Emergencies are unanticipated circumstances caused by accidents or harmful disasters that threaten the environment, properties, and people's lives. Regardless of the magnitude of the effect and whether emergencies occur completely unforeseen or are predictable, emergencies can only be managed if all the actors show more effective cooperation and coordination. Therefore, accurate and optimal provision of information is an essential prerequisite for crisis management and response to disasters since the decisions of any role-maker can have far-reaching implications. The variety of skills, abilities, and knowledge that the role-maker provides is essential to managing the crisis. However, this diversity is also regarded as one of the barriers since in a real emergency; all efforts must be directed toward a common goal. This approach may be valuable to any planner; however, it can lead to significant shortcomings in the cooperation and network systems of the command and control center $(12,21-23)$.

Communication and information exchange are of great importance in local, national, and global emergencies since the common shared infrastructures may be temporarily or even permanently inaccessible or unreliable. Due to the critical significance of communication and information exchange in an emergency and rescue processes (e.g., alerting, deployment, and distribution of facilities, and localization of resources needed for rescue processes), designing, and evaluating concepts for the use of advanced and pioneering technologies seem necessary. Regarding the nature of emergencies, the use of new technologies is essential to improve the logistics and required support to respond and reduce costs. The utilization of innovative blockchain technology in crisis management during emergencies can lead to cooperation among many parties involved in the response process, thereby creating transparency (23-26). Furthermore, in mass disasters with multinational victims, it is important to identify the deceased for judicial, ethical, religious, and human rights reasons, as well as allow relatives to complete the mourning process (27).

Disaster Victim Identification (DVI) is a complex process in which post-mortem identification (PM) data, mainly fingerprints, DNA, and teeth, are collected to match ante-mortem data (AM) in the list of missing individuals (26). Although there are ways to human identification, they all have flaws that usually do not lead to the identification of the human identity in a timely manner. Initially, the management of the list of missing people which involves years and sometimes decades, as well as updating the data sources of the AM family is very challenging. Additionally, there is currently no comprehensive technical solution for managing both AM and PM to identify humans that can support multinational and intergovernmental participatory processes (27, 28). Blockchain technology provides tools to facilitate the creation of reliable, secure, and comprehensive ecosystems that can publish AM 
and PM data across systems and protect against data breaches, redundancies, incompatibilities, and errors. Furthermore, this technology can revolutionize the process of identifying people around the world in terms of the management of missing people list, AM information resources for living people, and PM information resources for anonymous discovered victims; moreover, it compares compatible biological specifications for definitive identification. Therefore, using realworld scenarios, the researchers suggest a number of appropriate usages to gain a comprehensive understanding of the challenges and show how blockchain technology copes with such challenges and share information across multiple areas $(6,28-$ $31)$.

\section{Discussion and Conclusion}

Accurate and efficient provision of information is an essential prerequisite for crisis management and response to disasters since the decisions of any role-maker can have far-reaching implications. Innovative blockchain technology is extensively used in various fields and is usually very popular due to its decentralized and distributed organization and management (26-23). This study mainly aimed to investigate the framework for adopting blockchain technology in an emergency. This technology and its related knowledge can be utilized in the real world. According to the findings of the present study, it was shown that how blockchain technology meets all the requirements for trust and information security. Since blockchain can be specifically designed to fulfill the requirements of an information path among participants, it can be configured separately. Therefore, the entire multiblock architectures are solutions for controlling central systems, as well as connecting multiple commands and mobile emergency units.

Distributed and effective blockchain layout is more appropriate than integrated plans and resources due to the need for emergency scenarios. In particular, the need for timely, accurate, and reliable information appears to be met by blockchain technology .Other problems that can be emerged during and after the disaster include difficulties in finding the nearest relief center, spread of disease anger, power outages, uneven distribution of water and food, finding missing people, counting of injured people and damages, transportation, victim certification for future well-being, and fraudulent behavior to steal people's well-being. The blockchain has the potential to solve all these problems effectively without any delay.

According to the previously conducted studies, the use of emerging technologies other than traditional methods can be useful for physicians. Moreover, these technologies understand the challenges using science, and therefore, they are of great social and humanitarian importance and can affect entire societies and human rights $(6,28$ 31). In general, blockchain technologies have proven to be successful in reducing logistic, support, administrative, and management costs in different areas. Crisis management in emergencies and disasters can be significantly improved by the implementation of blockchain technologies. In addition, studies show that the implementation of blockchain technology in crisis management, emergency, and rescue processes is very complex and requires a great deal of cooperation from all parties; however, it will be effective in practice.

\section{Acknowledgments}

The authors would like to express their gratitude to all those who contributed to the conduction of this research project.

\section{Conflict of Interests}

Authors declared no conflict of interests regarding the publication of the present study.

\section{References}

1. Gao W, Hatcher WG, Yu W. A survey of blockchain: techniques, applications, and challenges. 27th International Conference on Computer Communication and Networks (ICCCN), Hangzhou, China; 2018. P. 1-11.

2. Zheng Z, Xie S, Dai H, Chen X, Wang H. An overview of blockchain technology: Architecture, consensus, and future trends. International Congress on Big Data (Big Data Congress), Honolulu, HI, USA; 2017. P. 557-64.

3. Izadi DS, Sharif-Khatibi Z. Blockchain and its usage in storing information as a secure distributed database. J Informat Communication Technol Polic 2020; 1(2): 85-106.

4. Siemon C, Rueckel D, Krumay B. Blockchain technology for emergency response. Proceedings of the 53rd Hawaii International Conference on System Sciences, Maui, Hawaii; 2020. P. 614-23.

5. Ali MS, Vecchio M, Pincheira M, Dolui K, Antonelli F, Rehmani MH. Applications of blockchains in the Internet of Things: a 
comprehensive survey. IEEE Communications Surveys Tutorials 2018; 21(2): 1676-717.

6. Alsalamah S, Nuzzolese E. Promising blockchain technology applications and use case designs for the identification of multinational victims of mass disasters. Front Blockchain 2020; 3: 34.

7. Yli-Huumo J, Ko D, Choi S, Park S, Smolander K. Where is current research on blockchain technology?-A systematic review. PLoS One 2016; 11(10): e0163477.

8. Aste T, Tasca P, Di Matteo T. Blockchain technologies: the foreseeable impact on society and industry. Computer 2017; 50(9): 18-28.

9. Burniske C, Vaughn E, Cahana A, Shelton J. How blockchain technology can enhance electronic health record operability. New York: Ark Invest; 2016.

10. Jovanovic B, Rousseau PL. General purpose technologies. Handbook of economic growth. New York: Elsevier; 2005.

11. Androulaki E, Barger A, Bortnikov V, Cachin C, Christidis K, De Caro A, et al. Hyperledger fabric: a distributed operating system for permissioned blockchains. Proceedings of the Thirteenth EuroSys Conference, Porto, Portugal; 2018.

12. Böhme R, Christin N, Edelman B, Moore $T$. Bitcoin: economics, technology, and governance. J Econ Perspect 2015; 29(2): 213-38.

13. Glaser F. Pervasive decentralization of digital infrastructures: a framework for blockchain enabled system and use case analysis. Frankfurt, Germany: Goethe University Frankfurt; 2017.

14. Seebacher S, Schüritz R. Blockchain technology as an enabler of service systems: a structured literature review. International Conference on Exploring Services Science, Rome, Italy; 2017.

15. Beck R, Avital M, Rossi M, Thatcher JB. Blockchain technology in business and information systems research. Bus Inf Syst Eng 2017; 59(6): 3814.

16. Lindman J, Tuunainen VK, Rossi M. Opportunities, and risks of Blockchain Technologies-a research agenda. Gothenburg, Sweden: University of Gothenburg; 2017.

17. Hölbl M, Kompara M, Kamišalić A, Nemec Zlatolas L. A systematic review of the use of blockchain in healthcare. Symmetry 2018; 10(10): 470.
18. Will blockchain transform healthcare? Forbes. Available at: URL: https://www.forbes.com/ sites/ciocentral/2018/08/05/willblockchaintransform-healthcare/2e63a56c553d; 2018.

19. Agbo CC, Mahmoud QH, Eklund JM. Blockchain technology in healthcare: a systematic review. Healthcare (Basel) 2019; 7(2): 56.

20. Pereira T, Morgado J, Silva F, Pelter MM, Dias VR, Barros R, et al. Sharing biomedical data: strengthening ai development in healthcare. Healthcare (Basel) 2021; 9(7): 827.

21. Black S, Hackman L. Disaster victim identification. Wiley encyclopedia of forensic science. New Jersey: John Wiley \& Sons; 2009.

22. Sakurai M, Murayama Y. Information technologies and disaster management-Benefits and issues. Progr Disaster Sci 2019; 2: 100012.

23. Sobha GV, Sridevi P. Usecase of blockchain in disaster management-a conceptual view. Greeley, Colorado: Aims Community College; 2019.

24. Erdelj M, Król M, Natalizio E. Wireless sensor networks and multi-UAV systems for natural disaster management. Comput Netw 2017; 124: 7286.

25. Cocco L, Pinna A, Marchesi M. Banking on blockchain: costs savings thanks to the blockchain technology. Future Internet 2017; 9(3): 25.

26. Khoo L, Soon Lai P, Hafizam Hasmi A, Shah Mahmood M. Secondary identifier for positive identification in DVI. Forensic Sci Criminol 2016; 1: $1-3$.

27. Voigt $\mathrm{P}$, Von dem Bussche A. The eu general data protection regulation (gdpr). A Practical Guide. Cham: Springer International Publishing; 2017.

28. Nuzzolese E. Missing people, migrants, identification and human rights. J Forensic Odontostomatol 2012; 30(Suppl 1): 47-59.

29. Irrera A. Accenture, Microsoft team up on blockchain-based digital ID network. New York: Reuters; 2017.

30. Morgan O, Tidball-Binz M, Van Alphen D. Management of dead bodies after disasters: a field manual for first responders. Washington DC: Pan American Health Organization (PAHO); 2006.

31. Yue X, Wang H, Jin D, Li M, Jiang W. Healthcare data gateways: found healthcare intelligence on blockchain with novel privacy risk control. J Med Syst 2016; 40(10): 1-8. 